\title{
Influência do tamanho de flocos e da concentração de ar dissolvido na eficiência da flotação
}

Influence of flocs size and dissolved air concentration on the efficiency of flotation

Andre Pioltine', Marco Antonio Penalva Reali²

口-

\section{RESUMO}

O presente artigo apresenta resultados de pesquisa que teve por objetivo a investigação da influência do tamanho de partículas floculadas na eficiência da clarificação de águas para abastecimento por flotação por ar dissolvido (FAD) como emprego de método e equipamento para a aquisição eanálise de imagens especialmente desenvolvidos para a caracterização da distribuição de tamanho de flocos e microbolhas de ar presentes no processo de flotação. Os ensaios foram realizados com água sintética (temperatura de $26,0 \pm 0,5^{\circ} \mathrm{C}$;

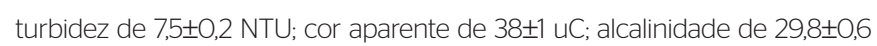

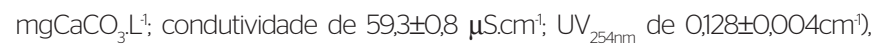

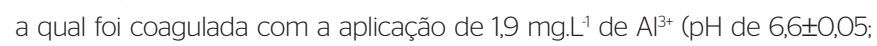
22,5 mg.L-1alum). Os resultados alcançados demonstraram que: i) a distribuição de tamanho de partículas floculadas influenciou diretamente a clarificação por flotação da água em estudo, sendo que as melhores condições de floculação foram aquelas que conduziram à formação, em sua maioria, de partículas floculadas com diâmetro médio de Feret acima de 300 m; ii) os menores valores residuais dos parâmetros analisados na água em estudo foram alcançados com o emprego da Condição 2 de floculação ( $\left(=17\right.$ min/G=60 s') associada a uma concentração em massa de ar $\left(C_{b}\right.$ ) de 4,8 mg. L-1, entretanto, a FAD foi capaz de operar também com boa eficiência com a utilização de tempo de floculação (T) de 10 minutos; iii) o método e o equipamento desenvolvidos nesta pesquisa para aquisição e análise de imagens demonstraram constituir importantes ferramentas para a obtenção das distribuições de tamanho de flocos e microbolhas de ar.

Palavras-chave: floculação; flotação por ar dissolvido; análise de imagens.

\begin{abstract}
This paper shows the results of a research that aimed to investigate the influence of flocculated particles size on the dissolved air flotation (DAF) efficiency. Special image acquisition method and equipment were developed to characterize the flocculated particles and air microbubbles size distribution in the contact zone outlet of the DAF unit. The trials were carried out with synthetic water (temperature of $26.0 \pm 0.5^{\circ} \mathrm{C}$; turbidity of $7.5 \pm 0.2 \mathrm{NTU}$; apparent color of $38 \pm 1 \mathrm{uC}$; alkalinity of $29.8 \pm 0.6 \mathrm{mgCaCO}_{3} . \mathrm{L}^{-1}$; conductivity of $59.3 \pm 0.8 \mu \mathrm{S} . \mathrm{cm}^{-1}$; $U_{254 \mathrm{~nm}}$ of $\left.0.128 \pm 0.004 \mathrm{~cm}^{-1}\right)$, that it was coagulated by applying $1.9 \mathrm{mg}^{-1} \mathrm{~L}^{-1}$ of $\mathrm{Al}^{3+}$ ( $\mathrm{pH}$ 6.6 $\left.\pm 0.05 ; 22.5 \mathrm{mg} . \mathrm{L}^{-1} \mathrm{alum}\right)$. The results showed that: i) the flocculation conditions interfered directly on the efficiency of DAF process, and the best flocculation conditions were those that conducted to formation of flocculated particles size with Feret mean diameter above $300 \mu \mathrm{m}$; ii) the best results were obtained applying Condition 2 of flocculation ( $\left.T=17 \mathrm{~min} / \mathrm{G}=60 \mathrm{~s}^{-1}\right)$ combined

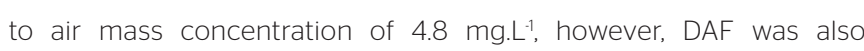
capable of operating with a satisfactory efficiency when applying flocculation time of 10 minutes; iii) the special image acquisition method and equipment developed proved to be an important tool to characterize the flocculated particles and air microbubbles size distribution.
\end{abstract}

Keywords: flocculation; dissolved air flotation; image analysis.

\section{INTRODUÇÃO}

Em vista do comprometimento acentuado da qualidade das águas dos mananciais superficiais, principalmente no que diz respeito ao aumento da concentração de substâncias orgânicas, os sistemas de flotação por ar dissolvido (FAD) têm sido cada vez mais utilizados em Estações de Tratamento de Água (ETAs) em todo o mundo, devido ao melhor desempenho dessa técnica em relação aos sistemas convencionais. Como exemplo, pode-se citar: ETA de Nova York, com capacidade de

$12,7 \mathrm{~m}^{3} \cdot \mathrm{s}^{-1}$ (CROSSLEY et al., 2007); ETA Sangjiancom, com capacidade de $2,3 \mathrm{~m}^{3} \cdot \mathrm{s}^{-1}$ (SOHN et al., 2007); ETA de Winnipeg, implantada no Canadá, com capacidade de $4,6 \mathrm{~m}^{3} \cdot \mathrm{s}^{-1}$ (PERNISTSKY et al., 2007). Especificamente no Brasil, já existe número expressivo de ETAs utilizando a flotação, podendo-se citar a ETA Brasília, no Distrito Federal $\left(2,8 \mathrm{~m}^{3} \cdot \mathrm{s}^{-1}\right)$, ETA Iraí, no Paraná $\left(4,0 \mathrm{~m}^{3} \cdot \mathrm{s}^{-1}\right)$, ETA Carapina, no Espírito Santo $\left(2,5 \mathrm{~m}^{3} \cdot \mathrm{s}^{-1}\right)$, e ETA Vargem das Flores, em Minas Gerais $\left(1,4 \mathrm{~m}^{3} \cdot \mathrm{s}^{-1}\right)$, todas elas já em operação.

'Engenheiro Ambiental. Professor Doutor do Centro Universitário Barão de Mauá - Ribeirão Preto (SP), Brasil.

Engenheiro Civil. Professor Doutor do Departamento de Hidráulica e Saneamento da Escola de Engenharia de São Carlos da Universidade de São Paulo (EESC/USP) - São Carlos (SP), Brasil. Endereço para correspondência: Andre Pioltine - Centro Universitário Barão de Mauá - Rua Ramos de Azevedo, 423 - Jardim Paulista - $14090-180$ - Ribeirão Preto (SP), Brasil E-mail: pioltine@gmail.com

Recebido em: 02/05/12 - Aceito em: 16/07/15 - Reg. ABES: 90030 
De acordo com Kiuru (2001), a FAD foi inicialmente utilizada no processamento de matérias-primas minerais no final do século XIX, sendo introduzida como alternativa para o tratamento de água na década de 1920. Durante as décadas de 1960 e 1970, a FAD foi extensivamente aplicada nos países escandinavos, como Suécia e Finlândia, no tratamento de água de abastecimento e águas residuárias devido à alta eficiência desse processo em águas frias na remoção de sólidos em suspensão formados pela coagulação e floculação de substâncias húmicas, especialmente quando comparado ao processo de sedimentação. Nos Estados Unidos, segundo Edzwald et al. (1999), a FAD começou a ganhar maior importância como pré-tratamento no final da década de 1990, após a promulgação da Surface Water Treatment Rule, a qual requereu de forma efetiva a utilização do processo de filtração no tratamento de águas superficiais. No Brasil, as primeiras pesquisas com o emprego da FAD no tratamento de águas de abastecimento iniciaram-se no início da década de 1980 na Escola de Engenharia de São Carlos da Universidade de São Paulo (EESC/USP).

Ao longo desses anos, importantes desenvolvimentos no processo de FAD foram verificados, contribuindo significativamente para o aumento da utilização dessa tecnologia tanto para o tratamento de águas de abastecimento quanto para o tratamento de efluentes industriais e domésticos. Dentre esses desenvolvimentos, Edzwald (2010) cita os importantes avanços ocorridos nos últimos 50 anos com relação ao aumento das taxas de aplicação superficial (de 5 para $40 \mathrm{~m} \cdot \mathrm{h}^{-1}$ ) empregadas no processo e a diminuição dos tempos de floculação aplicados na FAD (de 30 para 10 minutos). Além disso, nas últimas décadas diversos modelos matemáticos têm sido desenvolvidos e empregados, bem como diversas ferramentas, como análise de imagens, microADV (Accoustic Doppler Velocimetry), difração a laser, entre outros, os quais têm contribuído para uma melhor compreensão das interações ocorridas entre microbolhas de ar e flocos no interior de reatores FAD.

Entretanto, apesar desses avanços em relação ao conhecimento de diversos aspectos fundamentais do processo de FAD, pontos importantes permanecem pouco esclarecidos. Dessa forma, evidencia-se a necessidade da realização de estudos com o objetivo de aprofundar o nível de conhecimento acerca da cinética do processo de flotação, com ênfase na influência da distribuição de tamanho de flocos e microbolhas de ar na formação de aglomerados bolhas-floco.

Com relação às técnicas atualmente empregadas na captura e análise de imagens de flocos e microbolhas de ar formados durante o processo de flotação, tem se verificado que ao longo dos últimos anos ocorreu um aumento significativo no número de pesquisas desenvolvidas, dada a importância que essas partículas exercem sobre a cinética do processo de flotação (BACHE \& RASOOL, 2001; RODRIGUES \& RUBIO, 2003; MORUZZI, 2005; LEE et al., 2007; HAN et al., 2007; KWAK et al., 2009; SOHN et al., 2009; OLIVEIRA et al., 2010).

Dessa forma, o presente artigo apresenta resultados obtidos em pesquisa desenvolvida com o objetivo de investigar a influência do tamanho de partículas floculadas na eficiência da clarificação de águas para abastecimento por flotação com o emprego de método e equipamento para aquisição e análise de imagens especialmente desenvolvidos, tendo em vista a caracterização da distribuição de tamanho de flocos e microbolhas de ar.

\section{MATERIAL E MÉTODOS}

\section{Considerações gerais}

O presente estudo foi desenvolvido nos setores de Equipamentos de Bancada e de Instalações Piloto do Laboratório de Tratamento Avançado e Reuso de Águas (LATAR), pertencente ao Departamento de Hidráulica e Saneamento da EESC/USP.

Deve ser destacado que os valores de gradiente médio de velocidade de floculação $(G)$ empregados nos ensaios foram determinados experimentalmente por meio de sensor de torque especialmente adquirido e que as análises físico-químicas foram realizadas segundo as determinações do Standard Methods for the Examination of Water and Wastewater (2005).

\section{Água de estudo}

Com o objetivo de proporcionar um maior controle das condições experimentais, os ensaios foram realizados com água sintética preparada a partir da adição de $8,5 \mathrm{mg} . \mathrm{L}^{-1}$ de caulinita (marca FLUKA Analytical) e de 1,0 mg.L-1 de ácido húmico (marca ALDRICH Chemistry) à água proveniente de lençol subterrâneo localizado no Campus da EESC/USP.

A água sintética foi coagulada com a aplicação de $1,9 \mathrm{mg} \cdot \mathrm{L}^{-1} \mathrm{de} \mathrm{Al}^{3+}$ (22,5 mg.L L $^{-1}$ de sulfato de alumínio - marca VETEC P.A. 277). Para manter o pH na faixa ótima de coagulação $(6,6 \pm 0,05)$, foi utilizada solução de carbonato de sódio (marca GENCO). Estudo preliminar em unidade de flotação por batelada (equipamento denominado flotateste) foi realizado com objetivo de obter o $\mathrm{pH}$ e a dosagem ótima de coagulante para a água em estudo. Os resultados completos desse estudo preliminar podem ser obtidos nos trabalhos desenvolvidos por Araújo (2010) e Maia (2010).

É ressaltado que a água sintética empregada nesta pesquisa foi utilizada em outros estudos desenvolvidos no Departamento de Hidráulica e Saneamento da EESC/USP dentre linha mais ampla de pesquisa, sob a coordenação do Prof. Dr. Marco Antônio Penalva Reali, na qual o presente trabalho se insere. Dentre os estudos, podem ser citados os desenvolvidos por Reali e Patrizzi (2007), Moruzzi e Reali (2008), Araújo (2010), Maia (2010), Escher (2011) e Souza (2012).

A Tabela 1 apresenta as características da água sintética empregada nos ensaios.

\section{Unidade piloto de flotação}

A unidade piloto de FAD de escoamento contínuo empregada é constituída das seguintes partes principais: 
1. reservatórios de água com capacidade de $15 \mathrm{~m}^{3}$ cada, onde o ácido húmico (ALDRICH Chemistry) e a caulinita (FLUKA Analytical) são adicionados à água proveniente de lençol subterrâneo localizado no Campus da EESC/USP e misturados por agitadores submersos instalados no interior de cada um dos reservatórios;

2. aquecedores a gás in-line instalados paralelamente na alimentação de água sintética antes da unidade de mistura rápida;

3. unidade de mistura rápida tubular in-line responsável pela mistura rápida do coagulante;

4. compartimentos de floculação, nos quais os misturadores verticais são controlados eletronicamente operando na mesma rotação;

5. unidade de FAD retangular dividida em zona de contato e zona de separação;

6. câmara de saturação na qual a tubulação efluente é conectada a três válvulas do tipo agulha instaladas no fundo da zona de contato (sistema de despressurização);

7. sistema dosador para controle do processo de coagulação (bombas dosadoras e reservatórios para armazenamento de coagulante e alcalinizante);

8. bombas centrífugas associadas a medidores de escoamento eletromagnéticos e rotâmetros que alimentam a unidade piloto com água sintética e pressurizam o escoamento na câmara de saturação.

Todos os ensaios foram realizados com a utilização de dois estágios de floculação, ou seja, duas câmaras de floculação. É ressaltado que a diminuição do tempo de floculação $(\mathrm{T})$ empregado foi possível por meio do aumento da vazão afluente e da utilização de bomba de sucção instalada na parte inferior da segunda câmara de floculação, a qual ao succionar determinada vazão de água, que era descartada, permitia a operação da unidade piloto com os demais parâmetros operacionais da flotação constantes.

A Figura 1 apresenta a configuração da unidade piloto utilizada nos ensaios.

Tabela 1 - Características da água sintética.

\begin{tabular}{l|c} 
Parâmetro & Faixa de valores \\
\hline Temperatura $\left({ }^{\circ} \mathrm{C}\right)$ & $26,0 \pm 0,5$ \\
\hline Turbidez $(\mathrm{NTU})$ & $7,5 \pm 0,2$ \\
\hline Cor aparente $(\mathrm{UC})$ & $38 \pm 1$ \\
\hline Cor real $(\mathrm{uC})$ & $3,8 \pm 0,1$ \\
\hline Alcalinidade $\left(\mathrm{mgCaCO}_{3} \mathrm{~L}^{-1}\right)$ & $29,8 \pm 0,6$ \\
\hline Condutividade $\left(\mu \mathrm{S} . \mathrm{cm}^{-1}\right)$ & $59,3 \pm 0,8$ \\
\hline $\mathrm{UV} \mathrm{V}_{254 \mathrm{~nm}}\left(\mathrm{~cm}{ }^{-1}\right)$ & $0,128 \pm 0,004$ \\
\hline $\mathrm{pH}$ & $6,6 \pm 0,1$ \\
\hline
\end{tabular}

\section{Sistema de aquisição de imagens}

A caracterização da distribuição de tamanho de partículas floculadas (flocos) e microbolhas de ar foi realizada com equipamento especialmente desenvolvido, o qual foi composto por câmera CCD (charge-coupled device) especial de alta luminosidade (QImaging QICAM FAST MONO 12-bit) acoplada a um colposcópio (Olympus OCS-3) e a um microcomputador com software específico para captura e análise das imagens (Image-Pro Plus 7.01). A Figura 2 apresenta o esquema ilustrativo do sistema de aquisição e análise de imagens desenvolvido.

Deve ser ressaltado que o software Image-Pro Plus 7.01 foi utilizado por tratar-se de uma importante ferramenta para a aquisição e o processamento de imagens, possuindo diversas aplicações científicas, médicas e industriais, além de ter sido empregado em importantes pesquisas desenvolvidas por Kwak et al. $(2007,2009)$ e Sohn et al. (2009), as quais tiveram como objetivo a caracterização de flocos, microbolhas de ar e aglomerados bolhas-floco no processo de flotação.

Em relação ao colposcópio empregado, trata-se de um equipamento desenvolvido e utilizado na área médica, sendo que sua utilização foi por este apresentar distância focal ajustável de até $220 \mathrm{~mm}$, além de possuir uma fonte de luz intensa (150W) e elevada capacidade de ampliação (zoom de 7,9 até 23,5 x). É ressaltado que o acoplamento da câmera CCD de alta luminosidade ao colposcópio foi possível a partir de dispositivo especialmente desenvolvido para o presente trabalho.

Nos ensaios realizados, a aquisição das imagens das partículas floculadas e microbolhas de ar foi realizada por meio de sistema de sucção de amostras especialmente desenvolvido (sistema similar ao empregado por Leppinen e Dalziel, 2004). Esse sistema foi constituído por bomba, responsável pela sucção de amostras, e uma coluna retangular de amostragem (dimensões internas de $7 \times 3 \mathrm{~cm}$ e altura de $115 \mathrm{~cm}$ ) na qual era possível a aquisição de imagens. O desenvolvimento do sistema coluna de amostragem/bomba foi necessário devido à turbulência existente no interior da unidade piloto de flotação, a qual impossibilitava, durante os ensaios, a aquisição de imagens com qualidade suficiente para a análise posterior.

Durante os ensaios, a coluna de vidro (espessura de $4 \mathrm{~mm}$ ) era inserida verticalmente na saída da zona de contato do flotador (Figura 3) e pela bomba amostras de água contendo as partículas em estudo eram succionadas lentamente e visualizadas na coluna de amostragem, onde o sistema de aquisição estava instalado.

É ressaltado que no início da operação do sistema, após o preenchimento da coluna de amostragem, a velocidade no interior dessa coluna era ajustada a um valor igual à taxa de aplicação superficial empregada naquele momento na zona de contato $\left(\mathrm{TAS}_{\mathrm{ZC}}\right.$ ), com o objetivo de evitar o possível rompimento dos flocos. Após essa operação, a bomba era desligada e o fluxo de amostras era controlado pela abertura ou pelo fechamento de registro localizado na saída dessa coluna. No momento da aquisição de imagens, esse registro era lentamente fechado com o 
intuito de diminuir a velocidade das partículas e/ou bolhas no interior da coluna e favorecer a amostragem. Ressalta-se que toda vazão succionada era descartada durante a operação, não retornando ao tanque de flotação.
Parâmetro utilizado na caracterização do tamanho das partículas floculadas e microbolhas de ar

O tamanho das partículas floculadas e das microbolhas de ar foi obtido a partir do princípio da esfera equivalente na qual se utilizou
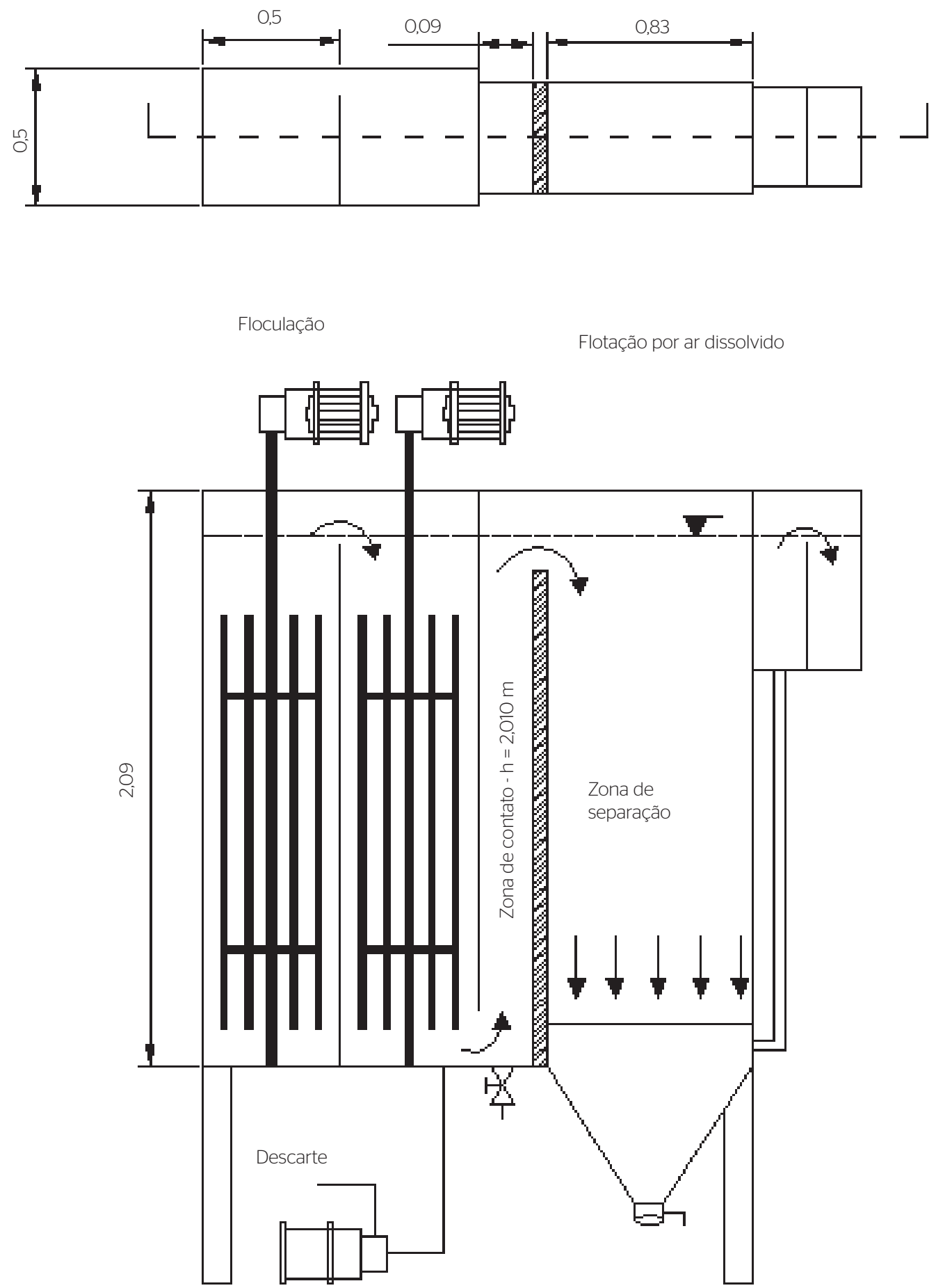

Figura 1 - Configuração da unidade piloto de flotação por ar dissolvido empregada; (A) planta; (B) corte (unidade em m). 
a medida linear denominada diâmetro de Feret. Esta medida consiste na distância paralela a uma direção fixa entre duas tangentes localizadas em lados opostos da partícula. Tal parâmetro é calculado diretamente pelo software Image-Pro Plus 7.01 (comando Feret Mean).

É ressaltado que esse parâmetro foi empregado em diversas pesquisas em que houve a caracterização de partículas (AL-THYABAT \& MILE, 2006; PABST et al., 2005; IGATHINATHANE et al., 2008; PARK et al., 2007; GRAU \& HEISKANEN, 2002).

\section{Programação dos ensaios}

Os ensaios foram realizados para duas condições de floculação: Condição $1-\mathrm{T}=10 \mathrm{~min} / \mathrm{G}=75 \mathrm{~s}^{-1}$, e Condição $2-\mathrm{T}=17 \mathrm{~min} /$

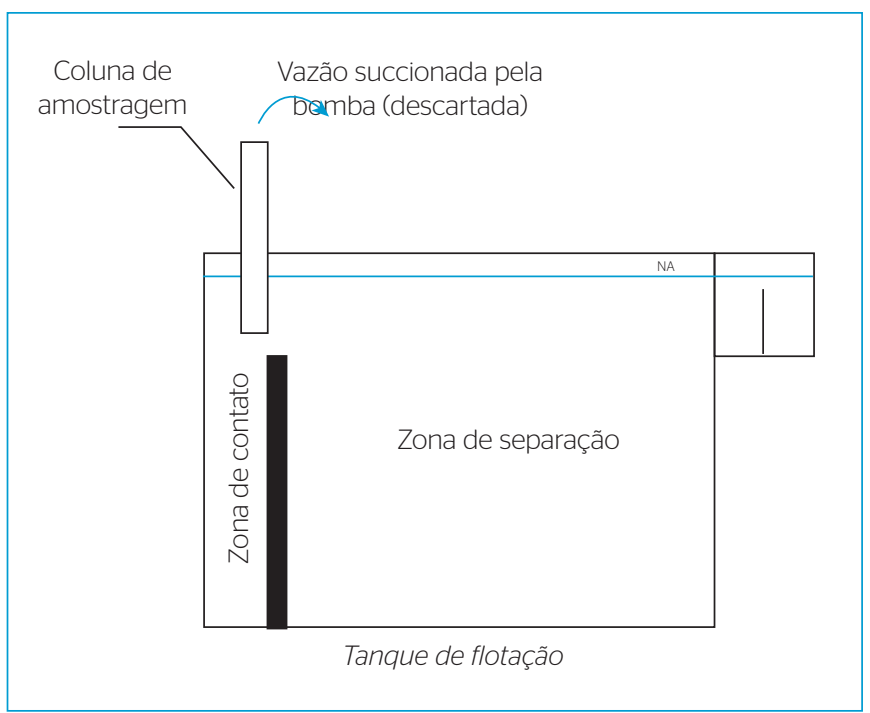

Figura 3 - Esquema ilustrativo do posicionamento da coluna de amostragem no interior do tanque de flotação durante o processo de aquisição das imagens.
$\mathrm{G}=60 \mathrm{~s}^{-1}$. É ressaltado que estas condições foram definidas com base nos menores valores de turbidez residual alcançados em ensaios (Ensaios Principais - Fase 2) realizados por Pioltine (2013). Todos os ensaios foram realizados em duplicata.

Para cada condição de floculação avaliada, diferentes valores de concentração em massa de ar $\left(\mathrm{C}_{\mathrm{b}}\right.$ igual a 3,3, 4,8 e 7,8 mg. $\mathrm{L}^{-1}$, relativas às razões de recirculação de 4,6 e 10\%, respectivamente) foram investigados.

Os parâmetros mantidos fixos durante todos os ensaios realizados estão apresentados na Tabela 2.

Tabela 2 - Parâmetros mantidos fixos durante todos os ensaios.

\begin{tabular}{|c|c|}
\hline \multicolumn{2}{|l|}{ Parâmetros fixos } \\
\hline Vazão afluente $\left(\mathrm{m}^{3} \cdot \mathrm{h}^{-1}\right)$ & $\begin{array}{l}3,6 \text { (T=17 minutos); } \\
\text { 6,2 ( } \mathrm{T}=10 \text { minutos) }\end{array}$ \\
\hline Temperatura da água afluente $\left({ }^{\circ} \mathrm{C}\right)$ & $26,1 \pm 0,5$ \\
\hline \multicolumn{2}{|l|}{ Mistura rápida } \\
\hline Dosagem de sulfato de alumínio/Al ${ }^{3+}\left(\mathrm{mg} \mathrm{L}^{-1}\right)$ & $22,5 / 1,9$ \\
\hline Dosagem de alcalinizante (mg.L'1) & 10 \\
\hline $\mathrm{pH}_{\mathrm{COAG}}$ & $6,6 \pm 0,05$ \\
\hline Potencial zeta (mV) & $-2,6 \pm 0,2$ \\
\hline \multicolumn{2}{|l|}{ Flotação por ar dissolvido } \\
\hline Velocidade de entrada na zona de contato $\left(m \cdot h^{-1}\right)$ & 84,0 \\
\hline Pressão de saturação (atm) & $4,5 \pm 0,1$ \\
\hline $\operatorname{TAS}_{\mathrm{zC}}\left(\mathrm{m} \cdot \mathrm{h}^{-1}\right)$ & $106,7 \pm 2,0$ \\
\hline $\mathrm{TDH}_{\mathrm{ZC}}(\mathrm{s})$ & $67,9 \pm 1,3$ \\
\hline Gradiente de velocidade na zona de contato $\left(s^{-1}\right)^{\star}$ & $1,9 \pm 0,05$ \\
\hline Velocidade cross-flow $\left(\mathrm{m} \cdot \mathrm{h}^{-1}\right)$ & $38,4 \pm 0,7$ \\
\hline $\operatorname{TAS}_{z S}\left(m \cdot h^{-1}\right)$ & $11,6 \pm 0,2$ \\
\hline Profundidade zona de separação (m) & 1,91 \\
\hline
\end{tabular}

*Valor estimado segundo equação utilizada por Richter (2009) para o cálculo do gradiente de velocidade em passagens submersas e utilizada no estudo desenvolvido por Maia (2010).

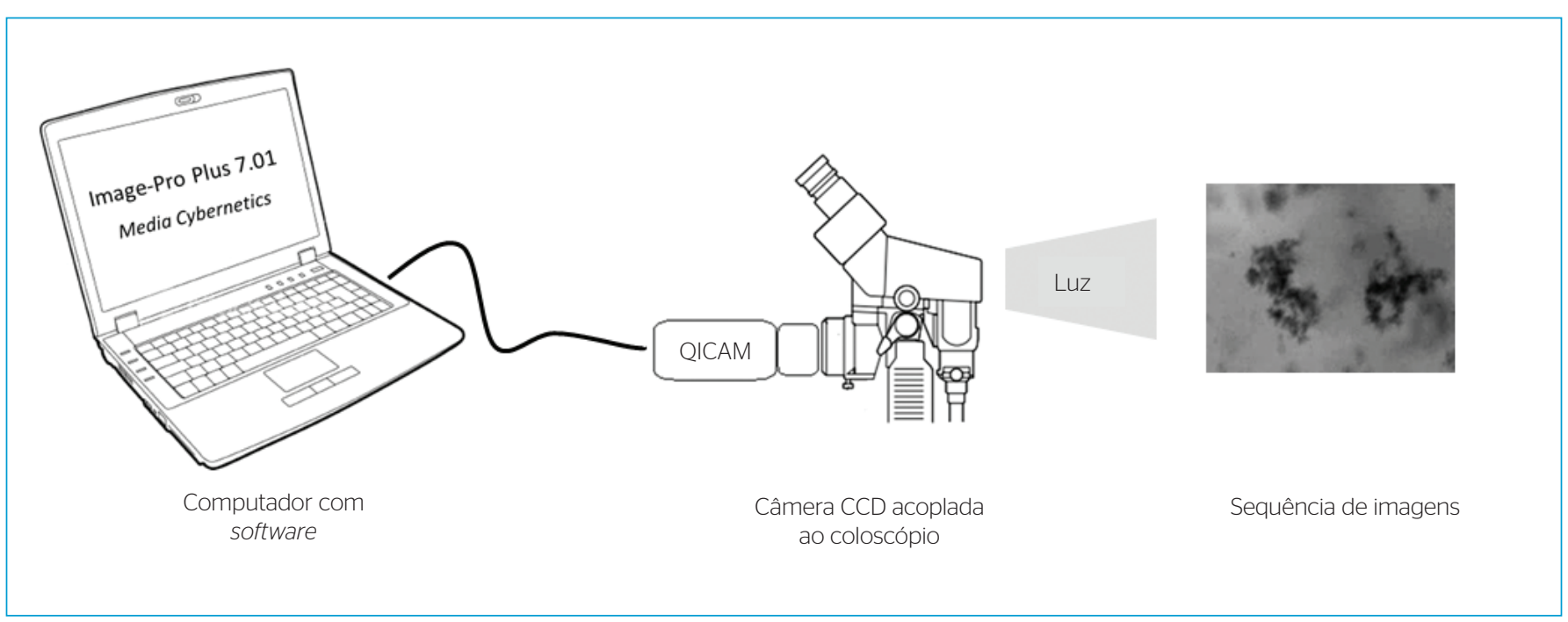

Figura 2 - Esquema ilustrativo do sistema de aquisição e análise de imagens desenvolvido para a aquisição de sequências de imagens de flocos e microbolhas de ar. 


\section{RESULTADOS E DISCUSSÃO}

A Figura 4 apresenta o efeito da $\mathrm{C}_{\mathrm{b}}\left(3,3,4,8\right.$ e $7,8 \mathrm{mg} \cdot \mathrm{L}^{-1}$, relativo às razões de recirculação de 4, 6 e 10\%) na FAD para as condições de floculação analisadas: Condição $1-\mathrm{T}=10 \mathrm{~min} / \mathrm{G}=75 \mathrm{~s}^{-1}$, e Condição 2 $\mathrm{T}=17 \mathrm{~min} / \mathrm{G}=60 \mathrm{~s}^{-1}$.

A partir dos gráficos apresentados na Figura 4, foi constatado que os menores valores residuais dos parâmetros analisados foram encontrados quando foi empregada a Condição 2 de floculação ( $\mathrm{T}=17 \mathrm{~min} / \mathrm{G}=60 \mathrm{~s}^{-1}$ ) associada a uma $\mathrm{C}_{\mathrm{b}}$ de $4,8 \mathrm{mg} \cdot \mathrm{L}^{-1}$ (turbidez de

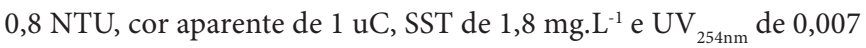
$\left.\mathrm{cm}^{-1}\right)$. Deve ser notado que o T que proporcionou os menores valores residuais dos parâmetros analisados encontra-se dentro da faixa de valores recomendada por diversos pesquisadores (normalmente na faixa de 10 a 20 minutos), conforme comentado por Edzwald (2010). De qualquer forma, faz-se necessária a realização de ensaios prévios em escala de laboratório e/ou escala piloto, com o objetivo de se determinar os valores de $\mathrm{T}$ e gradiente médio de $\mathrm{G}$ adequados ao tipo de água empregado.
Além disso, os resultados demonstraram que nos ensaios realizados na Condição 2 de floculação $\left(\mathrm{T}=17 \mathrm{~min} / \mathrm{G}=60 \mathrm{~s}^{-1}\right.$ ) associada à $\mathrm{C}_{\mathrm{b}}$ de 3,3 mg. $L^{-1}$, a quantidade de ar gerada foi insuficiente para promover uma adequada ascensão das partículas floculadas à parte superior do tanque de flotação e, consequentemente, houve uma baixa eficiência no processo de flotação. Fato oposto ocorreu nos ensaios realizados na Condição 1 ( $\mathrm{T}=10 \mathrm{~min} / \mathrm{G}=75 \mathrm{~s}^{-1}$ ) de floculação, em que foi verificada a obtenção de baixos valores residuais dos parâmetros analisados quando foi empregada $C_{b}$ de 3,3 mg. $L^{-1}$.

Tal fato foi provavelmente devido às diferentes distribuições de tamanho de flocos geradas nas duas condições de floculação analisadas, as quais são apresentadas na Figura 5.

A partir da distribuição do tamanho de partículas floculadas apresentada na Figura 5, foi verificado que a aplicação da Condição 1 de floculação ( $\mathrm{T}=10 \mathrm{~min} / \mathrm{G}=75 \mathrm{~s}^{-1}$ ) promoveu a formação de partículas, em sua maioria (em torno de $58 \%$ ), na faixa de 100 a $300 \mu \mathrm{m}$ de diâmetro médio de Feret, sendo que $62 \%$ das partículas geradas apresentaram diâmetro inferior a $300 \mu \mathrm{m}$, enquanto na Condição
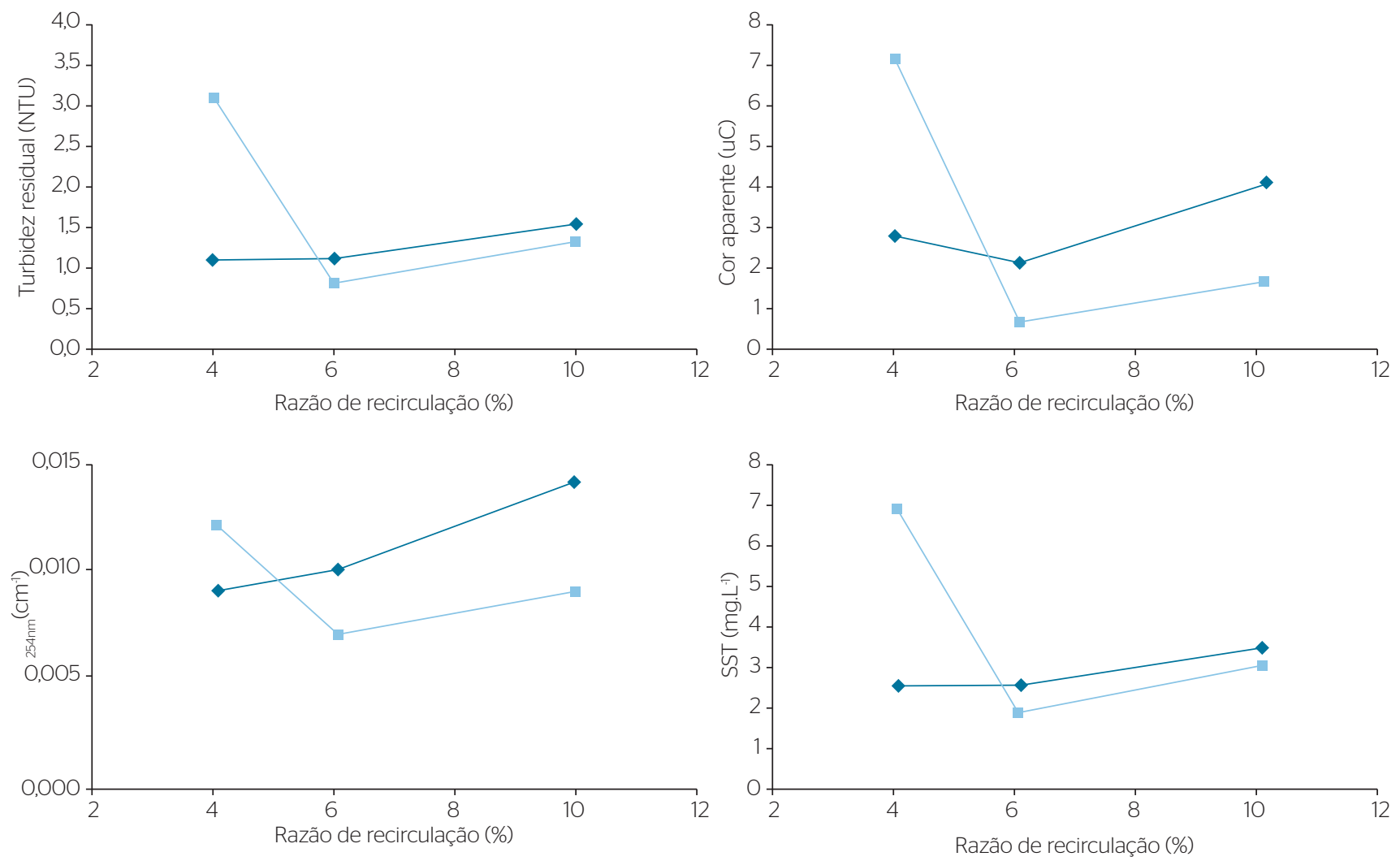

$T=10 \mathrm{~min} / \mathrm{G}=75 \mathrm{~s}^{-1}$

Figura 4 - Efeito da concentração em massa de ar na flotação por ar dissolvido para as condições de floculação analisadas: Condição 1 - T=10 min/ $\mathrm{G}=75 \mathrm{~s}^{-1}$, e Condição $2-\mathrm{T}=17 \mathrm{~min} / \mathrm{G}=60 \mathrm{~s}^{-1}$. 
2 de floculação ( $\mathrm{T}=17 \mathrm{~min} / \mathrm{G}=60 \mathrm{~s}^{-1}$ ), em que houve a formação de partículas, em sua maioria, na faixa de 300 a $600 \mu \mathrm{m}$ (em torno de $58 \%$ ), cerca de $30 \%$ das partículas apresentaram diâmetro inferior a $300 \mu \mathrm{m}$.

A Figura 6 apresenta exemplos de imagens de flocos obtidas com o emprego do sistema de aquisição e análise de imagens desenvolvido.
Nesses ensaios também pôde ser constatado que a FAD foi capaz de operar com boa eficiência quando foi empregada uma baixa $\mathrm{C}_{\mathrm{b}}\left(3,3 \mathrm{mg} \cdot \mathrm{L}^{-1}\right)$ associada à Condição 1 de floculação $\left(\mathrm{T}=10 \mathrm{~min} / \mathrm{G}=75 \mathrm{~s}^{-1}\right)$. Como comentado, nessa condição de floculação as partículas floculadas apresentaram, em sua maioria (em torno de 62\%), diâmetro médio de Feret inferior a $300 \mu \mathrm{m}$. Porém, é ressaltado que a eficiência obtida neste caso não foi

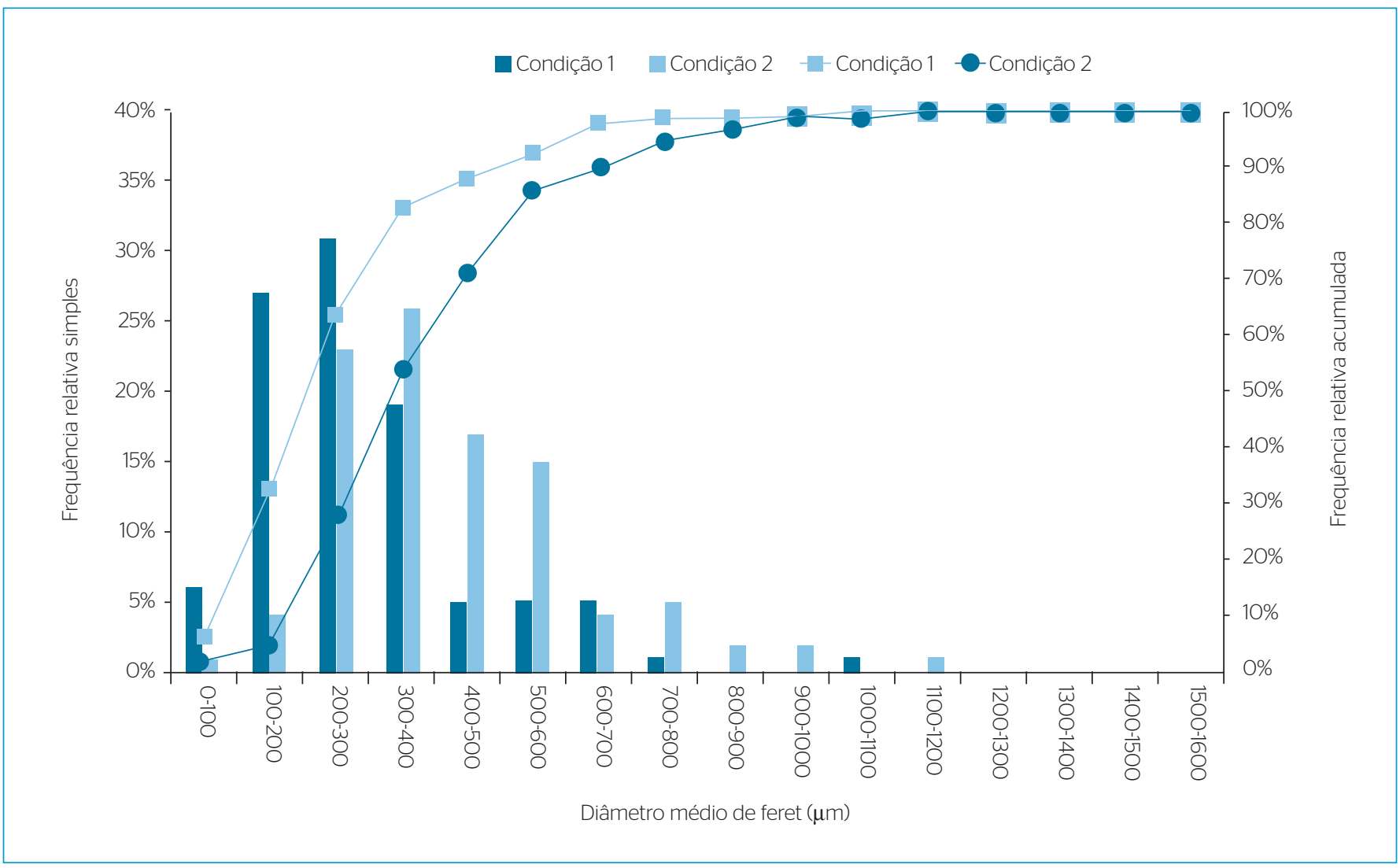

Figura 5 - Frequência relativa simples e acumulada em número por faixa de tamanho de partículas floculadas para as condições de floculação analisadas: Condição 1 - T=10 min/G=75 s-1, e Condição 2 - T=17 min/G=60 s-1.
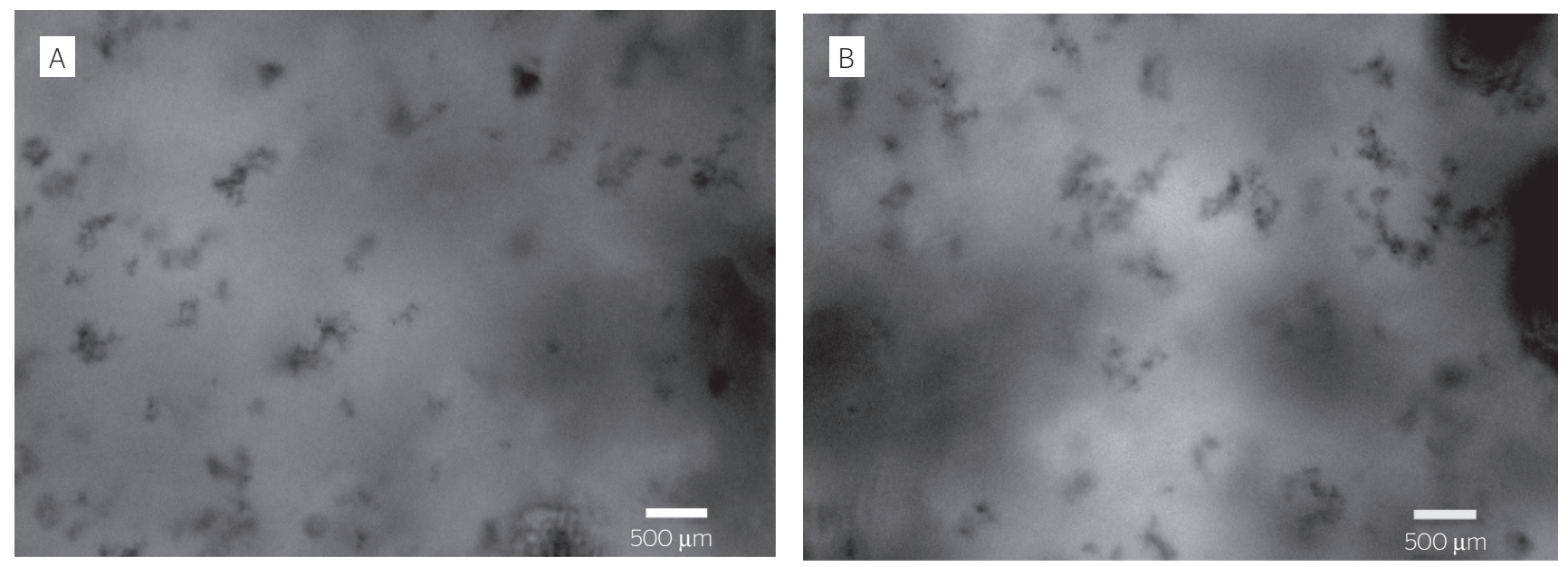

Figura 6 - Exemplos de imagens obtidas de flocos: Condição 1 ( $T=10 \mathrm{~min} / \mathrm{G}=75 \mathrm{~s}^{-1}-\mathrm{A}$ ) e Condição 2 ( $\mathrm{T}=17 \mathrm{~min} / \mathrm{G}=60 \mathrm{~s}-1$ - B). 
ótima, mas satisfatória e maior, quando comparada à eficiência obtida com a aplicação dessa mesma quantidade de ar na Condição 2 de floculação.

Com relação aos valores de $\mathrm{C}_{\mathrm{b}}$ investigados, como os demais parâmetros empregados na FAD foram mantidos fixos, observa-se que, à medida que se aumentou o valor de $\mathrm{C}_{\mathrm{b}}$ ( a partir de $3,3 \mathrm{mg} \cdot \mathrm{L}^{-1}$ ), houve aumento gradativo na taxa de aglutinação de microbolhas de ar no interior da zona de contato do tanque de flotação. Essa aglutinação, muito provavelmente, foi a responsável pela diminuição do desempenho da FAD nesses ensaios. Tal fato pôde ser comprovado a partir da realização de ensaios de aquisição e análise de imagens das microbolhas de ar geradas nas diferentes quantidades de $C_{b}$ investigadas, as quais são apresentadas na Figura 7.

Assim, a partir dos resultados apresentados na Figura 7 foi possível comprovar que o aumento na $\mathrm{C}_{\mathrm{b}}$, ou seja, o aumento na razão de recirculação (r), promoveu a aglutinação das microbolhas de ar, deslocando a curva de distribuição de tamanho de microbolhas de ar em direção às faixas de maior diâmetro. Dessa forma, quando foi empregada uma $\mathrm{C}_{\mathrm{b}}$ de 3,3 mg.L $L^{-1}$ (relativa à razão de recirculação de $4 \%$ e à pressão de saturação de 4,5 $\pm 0,1 \mathrm{~atm}$ ), a maior parte das microbolhas de ar (em torno de $79 \%$ ) apresentaram diâmetro entre 40 e $80 \mu \mathrm{m}$, enquanto o emprego de $\mathrm{C}_{\mathrm{b}}$ de $7,8 \mathrm{mg} . \mathrm{L}^{-1}$ (relativa à razão de recirculação de $10 \%$ e à pressão de saturação de 4,5 $\pm 0,1 \mathrm{~atm}$ ) proporcionou a formação de microbolhas de ar, em sua maioria, com diâmetro entre 80-120 $\mu \mathrm{m}$ (em torno de 55\%).
Em relação à curva de frequência relativa acumulada, foi verificado que para o emprego de $\mathrm{C}_{\mathrm{b}}$ de $3,3 \mathrm{mg} . \mathrm{L}^{-1}, 90 \%$ das microbolhas de ar apresentaram diâmetro com até $80 \mu \mathrm{m}$, enquanto com a utilização de $\mathrm{C}_{\mathrm{b}}$ de 7,8 mg. $\mathrm{L}^{-1}$, apenas $25 \%$ das microbolhas de ar tiveram diâmetro menor que $80 \mu \mathrm{m}$. Essa aglutinação das microbolhas de ar foi causada possivelmente pelo aumento da $\mathrm{C}_{\mathrm{b}}$ associado à taxa de aplicação superficial $\left(\mathrm{TAS}_{\mathrm{ZC}}\right.$ de $\left.106,7 \pm 2,0 \mathrm{~m} \cdot \mathrm{h}^{-1}\right)$ e tempo de detenção $\left(\mathrm{TDH}_{\mathrm{ZC}}\right.$ de $\left.67,9 \pm 1,3 \mathrm{~s}\right)$ aplicados na zona de contato do tanque FAD durante os ensaios.

A Figura 8 apresenta os valores médios de diâmetro de Feret obtidos para o emprego de $\mathrm{C}_{\mathrm{b}}$ de 3,3, 4,8 e 7,8 mg. $\mathrm{L}^{-1}$, incluindo o erro de estimativa calculado para um intervalo de confiança de 95\% (IC95\%).

Como pode ser observado na Figura 8, o aumento na $\mathrm{C}_{\mathrm{b}}$ promoveu aumento no diâmetro médio das microbolhas de ar, ou seja, ocorreu aglutinação destas microbolhas, como comentado.

A Figura 9 apresenta exemplos de imagens das microbolhas de ar obtidas com o emprego do sistema de aquisição e análise de imagens desenvolvido.

Em relação às interações ocorridas entre as microbolhas de ar, de acordo com Edzwald (2010), existem quatro forças que afetam o comportamento destas em termos de coalescência: força de London-van der Walls (atração), força eletrostática (repulsiva), interação hidrofóbica (atrativa) e repulsão hidrodinâmica (repulsiva). Segundo o autor, essas forças podem ser descritas

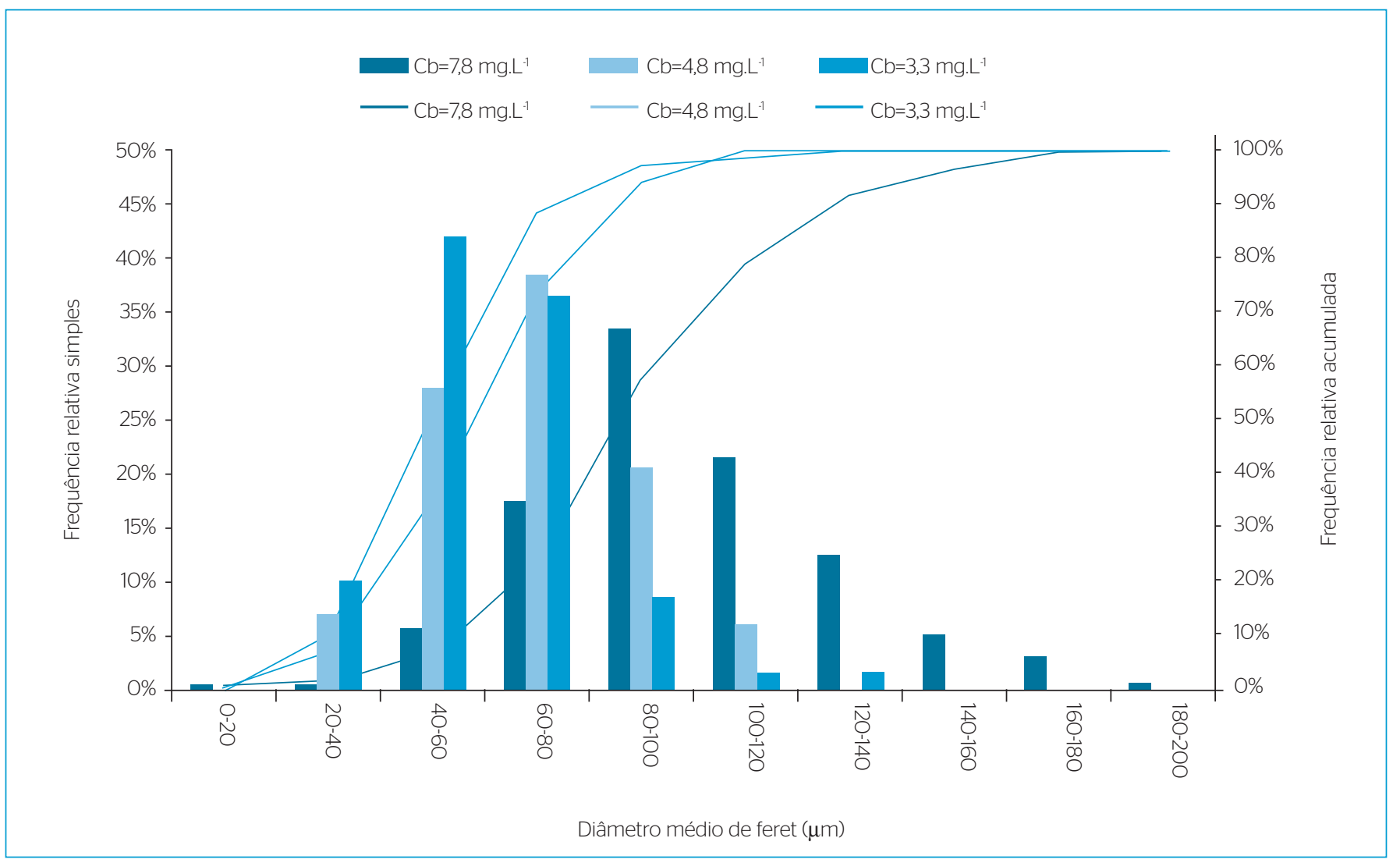

Figura 7 - Frequência relativa simples e acumulada em número por faixa de tamanho de microbolhas de ar para $\mathrm{Cb}$ de 7,8, 4,8 e 3,3 mg.L-1. 
conceitualmente, porém, sua quantificação é difícil. Além disso, Edzwald (2010) faz referência à pesquisa desenvolvida por Craig et al. * (1993, apud EDZWALD, 2010), na qual foi demonstrado que águas com baixas concentrações de sais favorecem a coalescência entre as bolhas de ar, enquanto em águas com altas concentrações de sais a coalescência praticamente não ocorre. Assim, como as águas utilizadas para o abastecimento possuem, em sua maioria, baixa concentração de sais (força iônica $<0,02 \mathrm{~mol} . \mathrm{L}^{-1}$ ), a coalescência entre as bolhas de ar é frequentemente constatada. De qualquer forma, para uma melhor compreensão dessas forças que afetam o comportamento das microbolhas de ar no interior dos tanques de FAD, a realização de um maior número de pesquisas é necessária, visto que o tamanho das microbolhas de ar influencia marcadamente a eficiência do processo de flotação, conforme verificado por diversos pesquisadores (KITCHENER \& GOCHIN, 1981; HAHN, 1984;ZABEL, 1984; RIJK et al., 1994; HAARHOFF \& EDZWALD, 2004; LEPPINEN \& DALZIEL, 2004; EDZWALD, 2007).

\section{CONCLUSÃO}

A distribuição de tamanho de partículas floculadas influenciou diretamente a clarificação por flotação da água em estudo, sendo que a

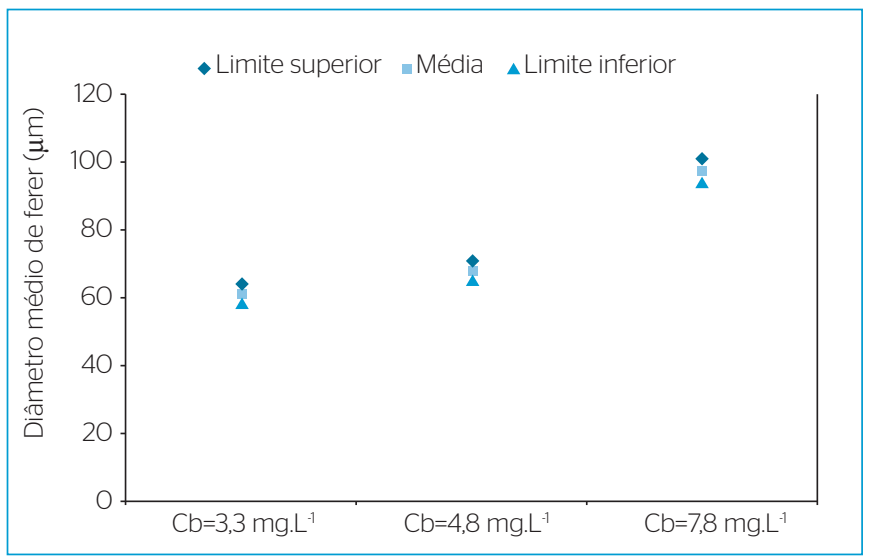

Figura 8 - Valores de diâmetro médio de Feret das microbolhas de ar incluindo intervalo de confiança de $95 \%$ para $\mathrm{Cb}$ de $3,3,4,8$ e 7,8 mg. $\mathrm{L}^{-1}$. melhor condição de floculação foi aquela que conduziu à formação, em sua maioria, de partículas floculadas com diâmetro médio de Feret acima de $300 \mu \mathrm{m}$ (Condição $2-\mathrm{T}=17 \mathrm{~min} / \mathrm{G}=60 \mathrm{~s}^{-1}$ ).

Os menores valores residuais dos parâmetros analisados na água em estudo foram alcançados quando foi empregada a Condição 2 de floculação ( $\mathrm{T}=17 \mathrm{~min} / \mathrm{G}=60 \mathrm{~s}^{-1}$ ) associada a uma $\mathrm{Cb}$ de $4,8 \mathrm{mg} \cdot \mathrm{L}^{-1}$ (turbidez de 0,8 NTU, cor aparente de $1 \mathrm{uC}$, SST de 1,8 mg.L $\mathrm{L}^{-1} \mathrm{e} \mathrm{UV}_{254 \mathrm{~nm}}$ de $\left.0,007 \mathrm{~cm}^{-1}\right)$. Entretanto, como pode ser verificado nos resultados apresentados, os valores de turbidez residual, cor aparente, SST e $\mathrm{UV}_{254 \mathrm{~nm}}$ encontrados na Condição 1 de floculação $\left(T=10 \mathrm{~min} / \mathrm{G}=75 \mathrm{~s}^{-1}\right)$ foram apenas ligeiramente superiores àqueles encontrados para a Condição 2, quando empregadas as situações de $\mathrm{Cb}$ de 4,8 e 7,8 mg.L $\mathrm{L}^{-1}$, e inferiores àqueles encontrados para a Condição 2 , quando empregada $\mathrm{a} \mathrm{Cb}$ de 3,3 mg. $\mathrm{L}^{-1}$, demonstrando que a FAD foi capaz de operar também com boa eficiência com a utilização de $\mathrm{T}$ de 10 minutos.

Nas condições de floculação analisadas, foi verificado que a aplicação de $\mathrm{T}=10 \mathrm{~min} / \mathrm{G}=75 \mathrm{~s}^{-1}$ (Condição 1) promoveu a formação de partículas, em sua maioria (em torno de 58\%), na faixa de 100 a 300 $\mu \mathrm{m}$ de diâmetro médio de Feret, sendo que $62 \%$ das partículas geradas apresentaram diâmetro inferior a $300 \mu \mathrm{m}$. Por outro lado, a aplicação da melhor condição de ensaio (Condição $2-\mathrm{T}=17 \mathrm{~min} / \mathrm{G}=60$ $\left.\mathrm{s}^{-1}\right)$, proporcionou a formação de partículas, em sua maioria, na faixa de 300 a $600 \mu \mathrm{m}$ (em torno de 58\%), com cerca de 30\% das partículas apresentando diâmetro inferior a $300 \mu \mathrm{m}$.

O método e o equipamento desenvolvidos para aquisição e análise de imagens demonstraram constituir importantes ferramentas para a obtenção das distribuições de tamanho de flocos e microbolhas de ar gerados no processo de flotação.

A partir dos resultados alcançados, recomendam-se, para projetos futuros de unidades de FAD utilizadas no tratamento de águas para abastecimento com características similares à água sintética empregada nesta pesquisa, o emprego de valores de gradiente médio de $\mathrm{G}$

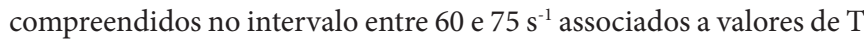
superiores a 10 minutos e à $\mathrm{C}_{\mathrm{b}}$ de $4,8 \mathrm{mg} \cdot \mathrm{L}^{-1}$.
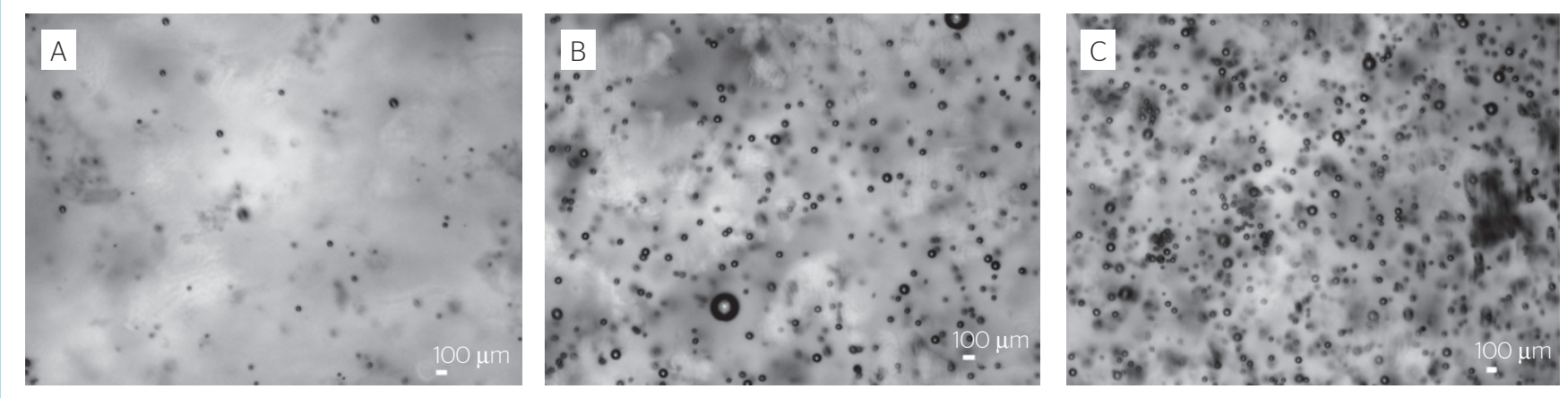

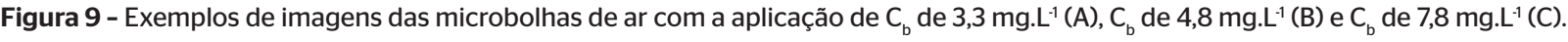

*CRAIG, V.S.J.; NINHAM, B.W.; PASHLEY, R.M. (1993) Effect of electrolytes on bubble coalesence. Nature, v. 364, p. 317-319. 


\section{REFERÊNCIAS}

AL-THYABAT, S. \& MILES, N.J. (2006) An improved estimation of size distribution from particle profile measurements. Powder Technology, v. 166 , p. $152-160$.

ARAÚJO, C.J. (2010) Influência da velocidade cross-flow na câmara de flotação na eficiência de unidades de flotação por ar dissolvido tratando água para abastecimento. Dissertação (Mestrado em Hidráulica e Saneamento) - Escola de Engenharia de São Carlos, Universidade de São Paulo, São Carlos.

BACHE, D.H. \& RASOOL, E. (2008) Characteristics of alumino-humic flocs in relation to DAF performance. Water Science and Technology, v. 43 , n. 8 , p. $203-208$.

CROSSLEY, I.A.; HERZNER, J.; BISHOP, S.L.; SMITH, P.D. (2OO7) Going Underground - Constructing New York City 's First Water Treatment Plant, a 1,100ml/d Dissolved Air Flotation, Filtration an UV Facility. In: The $5^{\text {th }}$ International Conference on Flotation in Water and Wastewater, Seoul. Proceedings the 5th International Conference on Flotation in Water and Wastewater Systems, v. 1, p. 141-152.

EATON, A.D.; CLESCERI, L.S.; RICE, E.W.; GREENBERG, A.E. (Eds.). (2005) APHA Standard Methods for the Examination of Water and Wastewater. $21^{\text {st }}$ ed. Washington: American Public Health Association.

EDZWALD, J.K.; WALSH, J.P.; KAMINSKI, G.S.; DUNN, H.J. (1992) Floculation and air requirements for dissolved air flotation. Journal of American Water Works Association - AWWA, v. 84, n. 3, p. 92-100

EDZWALD, J.K.; TOBIASON, J.E.; AMATO, T. MAGGI, L.J. (1999) Integrating high-rate DAF technology into plant design. Journal of American Water Works Association - AWWA, v. 91, n. 12, p. 41-53.

EDZWALD, J.K. (2007) The science and engineering of dissolved air flotation for drinking water treatment. In: The $5^{\text {th }}$ International Conference on Flotation in Water and Wastewater Systems, Seul. Proceedings the 5th International Conference on Flotation in Water and Wastewater Systems, Seoul.

ESCHER, A.E. (2011) Influência da taxa de aplicação superficial e da concentração de arna eficiência de clarificação deágua de abastecimento em unidades de flotação por ar dissolvido. Dissertação (Mestrado em Ciências, Programa de Engenharia Hidráulica e Saneamento) - Escola de Engenharia de São Carlos, Universidade de São Paulo, São Carlos.

GRAU, R.A. \& HEISKANEN, K. (2002) Visual technique for measuring bubble size in flotation machines. Minerals Engineering, v. 15, p. 507-513.

HAHN, H. Wastewater Treatment. In: IVES, K.J. (Ed.) (1984) The Scientific Basis of Flotation. Boston, USA: NATO ASI Series, Martinus Nijhoff. p. 379-413.

HAN, M.; KIM, T.; KIM, J. (2007) Effects of floc and bubble size on the efficiency of the dissolved air flotation (DAF) process. Water Science and Technology, v. 56, n. 10, p. 109-115.

HAARHOFF, J. \& EDZWALD, J.K. (2004) Dissolved air flotation modeling: insights and shortcomings. Journal of Water Supply: Research and Technology - AQUA, v. 53, n. 3, p. 127-150.
IGATHINATHANE, C.; PORDESIMO, L.O.; COLUMBUS, E.P.; BATCHELOR, W.D.; METHUKU, S.R. (2008) Shape identification and particles size distribution from basic shape parameters using Image. Computers and electronics in agriculture, v. 63, p. 168-182.

KITCHENER, J.A. \& GOCHIN, R.J. (1981) The mechanism of dissolved air flotation for potable water: basic analysis and a proposal. Water Research, v. 15, p. 585-590.

KWAK, D.H.; JUNG, H.J.; LEE, J.W.; KWON, S.B.; KIM, S.J.; YOO, S.J.; WON, C.H. (2007) Rise velocity verification of bubble-floc agglomerates using population balance in the DAF process. In: The $5^{\text {th }}$ International Conference on Flotation in Water and Wastewater Systems, Seoul. Proceedings the 5th International Conference on Flotation in Water and Wastewater Systems. v. 1. p. 121-134.

KWAK, D.H.; JUNG, H.J.; KWON, S.B.; LEE, E.J.; WON, C.H.; LEE, JW.; YOO, S.J. (2009) Rise velocity verification of bubble-floc agglomerates using population balance in the DAF process. Journal of Water Supply: Research and Technology - AQUA, v. 58, n. 2, p. 85-94.

KIURU, H.J. (2001) Development of dissolved air flotation technology from the first generation to the newest (third) one (DAF in turbulent flow conditions). Water Science and Technology, v. 43, n. 8, p. 1-7.

LEE, B.; HA, J.; PARK, J.; YANG, H. (2007) Limiting Size of a Particle or a Floc for Flotation in the DAF (Dissolved Air Flotation) Process. In: The $5^{\text {th }}$ International Conference on Flotation in Water and Wastewater Systems, Seoul. Proceedings the 5th International Conference on Flotation in Water and Wastewater Systems.

LEPPINEN, D.M. \& DALZIEL, S.B. (2004) Bubble size distribution in dissolved air flotation tanks. Journal of Water Supply: Research and Technology - AQUA, v. 53, p. 531-543.

MAIA, K.A. (2010) Influência da velocidade de entrada na zona de contato na eficiência de clarificação de unidades retangulares de flotação por ar dissolvido aplicado no tratamento de águas para abastecimento.. Dissertação (Mestrado em Hidráulica e Saneamento) - Escola de Engenharia de São Carlos, Universidade de São Paulo, São Carlos.

MORUZZI, R.B. \& REALI, M.A.P. (2008) Investigação da coagulação e floculação adequadas para a FAD no tratamento de água com cor aparente moderada e baixa turbidez destinada ao abastecimento público [Internet]. Teoria e Prática na Engenharia Civil, v. 8, n. 11, p. 55-66. Open Access. [cited 2015 Mar 17]. Available from: http://www. editoradunas.com.br/revistatpec/Art6_N11.pdf

MORUZZI, R.B. (2005) Flotação por ar dissolvido (FAD): Influência da distribuição de tamanho de partículas e do binômio velocidade/tempo de detenção na zona de reação no desempenho do processo. Tese (Doutorado em Hidráulica e Saneamento) - Escola de Engenharia de São Carlos, Universidade de São Paulo, São Carlos.

OLIVEIRA, C.; RODRIGUES, R.T.; RUBIO, J. (2010) A new technique for characterizing aerated flocs in flocculation-microbubble flotation system. International Journal of Mineral Processing, v. 96, p. 36-44. 
PABST, W.; BERTHOLD, C.; GREGOROVÁ, E. (2005) Size and shape characterization of polydisperse short-fiber systems. Journal of the European Ceramic Society, v. 26, p. 1121-1130.

PARK, S.; KIM, T.; KIM, Y. (2007) Effects of particle properties and bubble size on the efficiency of dissolved air flotation. In: The $5^{\text {th }}$ International Conference on Flotation in Water and Wastewater Systems, Seoul. Proceedings the 5th International Conference on Flotation in Water and Wastewater Systems.

PERNITSKY, S.; BREESE, S.; WOBMA, P.; GRIFFIN, D; KJARTANSON, K. SOROKOWSKY, R. (2007) From pilot tests to design on Canada's largest DAF water treatment plant. In: The $5^{\text {th }}$ International Conference on Flotation in Water and Wastewater, 2007, Seoul. Proceedings the 5th International Conference on Flotation in Water and Wastewater Systems. v. 1, p. 203-210.

PIOLTINE, A. (2013) Influência do tamanho de partículas floculadas na eficiência da pré-clarificação de águas para abastecimento por flotação. Tese (Doutorado em Ciências, Programa de Engenharia Hidráulica e Saneamento) - Escola de Engenharia de São Carlos, Universidade de São Paulo, São Carlos.

REALI, M.A.P. \& DOMBROSKI, S.A.G. (1996) Influência da floculação sobre a flotação por ar dissolvido. In: Congreso Interamericano de Ingenieria Sanitária y Ambiental -96, Cidade do México, 25 Anais. Cidade do México: AIDIS. v. 1. p. disco.

REALI, M.A.P. \& PATRIZZI, L.J. (2007) The influence of contact zone configuration on the color and turbidity removal efficiencies of a dissolved air flotation pilot plant In: The 5th International Conference in Flotation in Water and Wastewater Systems, 2007, Seoul. Proceedings the $5^{\text {th }}$ International Conference on Flotation in Water and Wastewater Systems. v. 1, p. 135-142.
RIJK, S.E.; VAN DEER GRAAF, J.H.J.M.; BLANKEM, J.G. (1994) Bubble size in flotation thickening. Water Research, v. 28, n. 2, p. 465-473.

RICHTER, C.A. (2009) Água: Métodos e Tecnologia de Tratamento. 1 ed. São Paulo: Editora Blucher. 340 p.

RODRIGUES, R.T. \& RUBIO, J. (2003) New basis for measuring the size distribution of bubbles. Minerals Engineering, v. 16, p. 757-765.

SOUZA, I.M.F. (2012) Influência do gradiente médio de velocidade na zona de contato na eficiência de clarificação de água para abastecimento por flotação. Dissertação (Mestrado em Ciências, Programa de Engenharia Hidráulica e Saneamento) - Escola de Engenharia de São Carlos, Universidade de São Paulo, São Carlos.

SOHN, B.Y.; PARK, T.J.; KWON, S.B.; OH, B.S.; KANG, J.W. (2007) A case study for process optimization of a DAF plant in Korea. In: The $5^{\text {th }}$ International Conference on Flotation in Water and Wastewater, Seoul. Proceedings the $5^{\text {th }}$ International Conference on Flotation in Water and Wastewater Systems, v. 1, p. 183-190.

SOHN, B.Y.; KWON, S.B.; MIN, J.H.; KANG, J.W. (2009) Characteristics of pretreatment parameters and bubble size influencing DAF efficiency. Separation Science and Technology, v. 44, p. 1941-1953.

VALADE, M.T.; EDZWALD, J.K.; TOBIASON, J.E.; DAHLQUIST, J.; HEDBERG, T.; AMATO, T. (1996) Particle removal by flotation and filtration: pretreatment effects. Journal of American Water Works Association, v. 88, n. 12, p. 35-47.

ZABEL, T. Flotation in Water Treatment. In: IVES, K.J. (Ed.) (1984) The Scientific Basis of Flotation. Boston: NATO ASI Series. p. 379413. 